\title{
AI-based Feature Detection in X-ray-CT Images Using Synthesized Data
}

\author{
Matthew Konnik ${ }^{1}$, Bahar Ahmadi $^{2}$, Nicholas May ${ }^{3}$, Joseph Favata ${ }^{4}$, Zahra Shahbazi ${ }^{5}$ Sina \\ Shahbazmohamadi ${ }^{4}$ and Pouya Tavousi ${ }^{4}$ \\ ${ }^{1}$ University of Illinois Champaign-Urbana, Urbana, Illinois, United States, ${ }^{2}$ UCONN/REFINE lab, \\ Vernon Rockville, Connecticut, United States, ${ }^{3}$ REFINE lab/ UCONN, Storrs, Connecticut, United States, \\ ${ }^{4}$ University of Connecticut, Storrs, Connecticut, United States, ${ }^{5}$ Manhattan College, Riverdale, New \\ York, United States
}

Nondestructive volumetric analysis of samples, enabled by X-ray computed tomography (CT), has attracted scientists and engineers from a wide spectrum of disciplines that are interested in identification and measurement of miniature internal features of their samples [1]. While obtaining X-ray CT images of arbitrary objects has become a straightforward procedure, which only requires adjustment of a few imaging parameters (e.g., energy, \# of projections), the interpretation of the resulting 3D images is still a challenging task [2]. For proper interpretation of an X-ray CT image, one must be able to extract welldefined geometric features from the raw data, where the raw data is a gray-scale 3D array of voxels [3]. Conventionally this task is performed manually by the subject matter experts (SMEs). Th extensive time and effort as well as human error associated with manual processes call for automated methods that can extract features accurately and with a high-throughput. The most common approach for achieving this goal is use of computer-vison (CV) techniques, to segment the images into distinct partitions [6] [7] [8] [9] [10] [11] [12] [13] [14], which could hopefully be used for extracting meaningful geometric features. For example, in thresholding, a common CV technique, intensity values and a preset thresholding constant will be used to assign a label to each pixel (voxel) in the 2D (3D) image. Such label is shared among all the pixels (voxels) of the same partition and the result of the segmentation process is a 2D (3D) image that is partitioned into several groups of connected pixels (voxels). Although, the CV techniques may offer an automated process in the absence of image noise (i.e., features that are of no interest), their performance drops drastically in dealing with noise which is prevalent in any image obtained from an X-ray CT practice [15] [16] [17]. The produced noise can be mitigated, but not completely removed. Therefore, in practice, the CV methods are only used to assist the manual feature extraction process and cannot provide a fully automated feature extraction process. The success of machine learning (ML) algorithms in automating tasks that are not analytically well-defined, promises use of these methods for automated feature extraction, as a superior alternative to $\mathrm{CV}$-based methods. The idea is to train a machine learning algorithm with sufficient ground truth data [18] [19] [20] and then use it for automated feature extraction. Here, the ground truth data are obtained from labeled X-ray CT images. Each data point consists of: (1) raw data in the form of a gray-scale 3D array of voxels and (2) the corresponding feature. The caveat is that the proper training of a machine learning algorithm demands huge amounts of labeled data. This is a multifold challenge. The necessity of labeling the raw data manually, makes this process extremely tedious, if not impractical. In addition, labeling process will be subjective, with different outcomes expected from different SMEs. Further, such manual process is subject to error. Double-checking and triple-checking practices to eliminate such error would add to the required time and effort for conducting the manual segmentation. Existing attempts to crowdsource the manual labeling task using volunteers [21] [22] [23] face the challenge of volunteer's lack of motivation. Also, a major drawback of the state-of-the-art software methods that enable manual segmentation is that the user, at each point in time, has only a 2D perception of the sample, which acts as a prohibitive factor on the way of conducting a realistic feature extraction, given than features extend in three dimensions. Moreover, the data that can be naturally found 
in the sample images might not be sufficient or diverse enough for training the machine learning algorithms. We propose to address these issues, through imaging of synthesized features. In this process, for any application, depending on the expected classes of features, certain geometries will be modeled in silico and in a parametric fashion. For example, for studying the electrical connections of a multi-layer PCB board, the parametric model of wirings and connections will be generated. Next, through a combinatorial algorithm, different sets of random values will be assigned to the feature parameters to enumerate a large set of features of different shapes, sizes, positions and orientations. This is followed by embedding the computer-synthesized features into the physical sample. Two key considerations in doing so are: (1) the features must be internal rather than appearing on the surface of the object to suit the application of X-ray CT; and (2) the geometric size of these features must be comparable to the naturallyfound instance of these features. To satisfy the first condition, we adopted a two-step solution: (a) create features on thin layer objects; and (b) stack the layers to form a 3D object. To satisfy the second condition, we employed the femtosecond pulsed laser which enabled us to fabricate distinct features down to tens of micrometers. The resulting 3D object would then undergo an X-ray CT process. The resulting image of each feature will be paired with the known geometric parameters that were used as a recipe for machining that feature. The resulting pair will be one of many training data points in the entire training data set. Taking the proposed approach compensates for the shortage and lack of diversity of naturally found data. Furthermore, since the exact geometry, position and orientation of each feature is known a priori, the proposed approach offers a significant improvement to the manually labeled data in terms of accuracy and robustness. Finally, needless to say, this approach can be fully automated. 

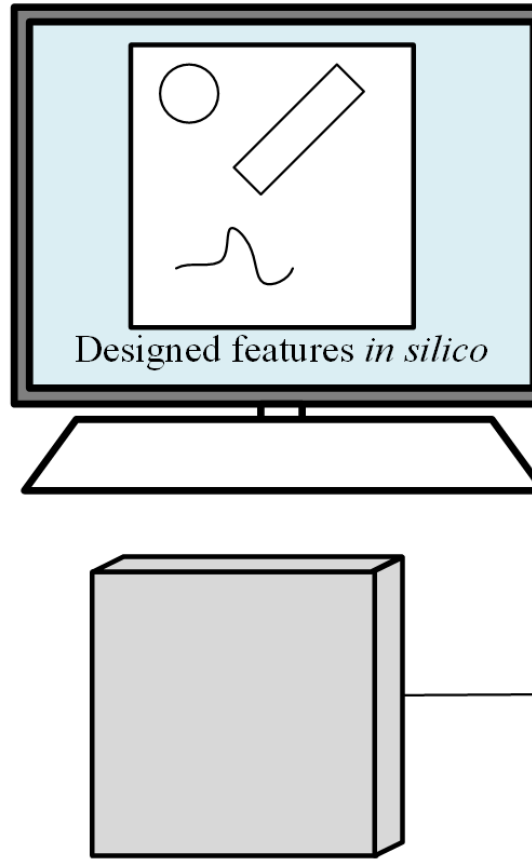

Thin layer

Embedded

after designed features embedding features

Thin layer with no features
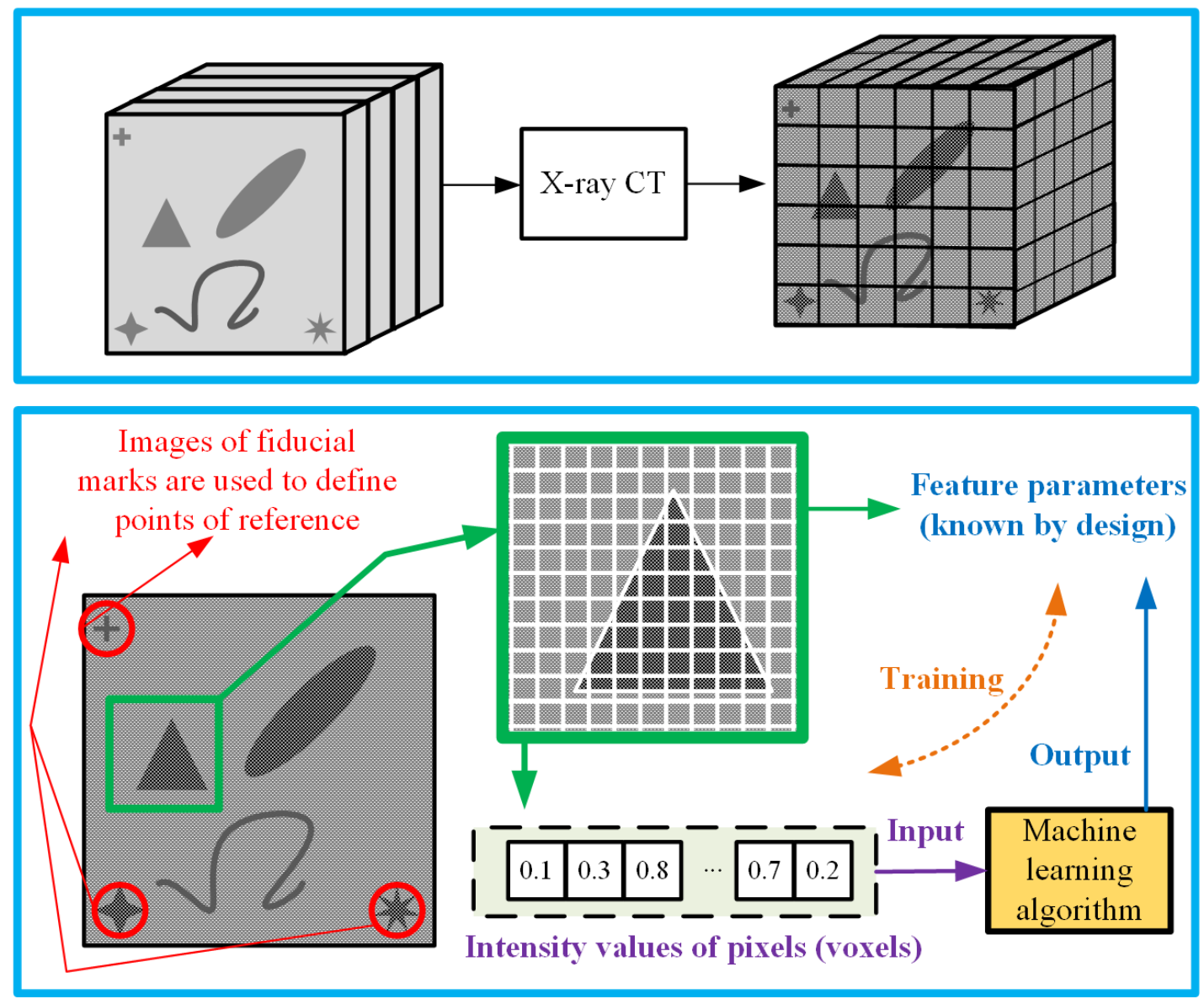

Figure 1. Schematic description of the proposed method 

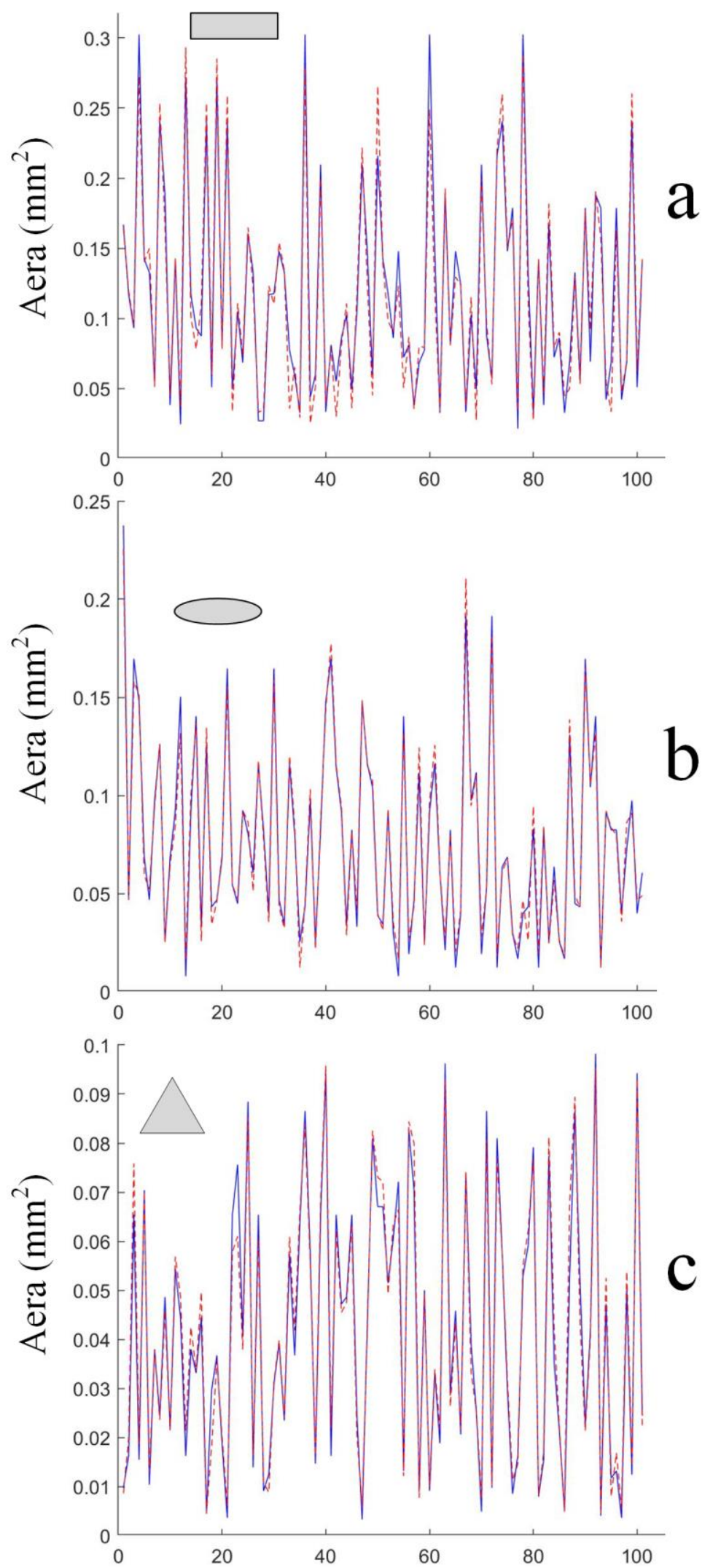

Figure 2. Areas of features predicted by ANNs. Solid blue indicates truth and dotted red indicates network prediction: (a) rectangles; (b) ellipses; (c) triangles. 


\section{References}

[1] J. M. Ingman, J. P. A. Jormanainen, A. M. Vulli, J. D. Ingman, K. Maula, T. J. Kärkkäinen and P. Silventoinen, "Localization of dielectric breakdown defects in multilayer ceramic capacitors using 3D Xray imaging," Journal of the European Ceramic Society, vol. 39, pp. 1178-1185, 2019.

[2] G. Wang, H. Yu and B. De Man, "An outlook on x-ray CT research and development," Medical physics, vol. 35, pp. 1051-1064, 2008.

[3] Y. Wei, H. Yu, J. Hsieh, W. Cong and G. Wang, "Scheme of computed tomography," Journal of XRay Science and Technology, vol. 15, pp. 235-270, 2007.

[4] D. Opitz and S. Blundell, "Object recognition and image segmentation: the Feature Analyst ${ }^{\circledR}$ approach," in Object-based image analysis, Springer, 2008, pp. 153-167.

[5] T. Blaschke and S. Lang, "Object based image analysis for automated information extraction--a synthesis," in Measuring the Earth II ASPRS Fall Conference, 2006.

[6] R. C. Gonzalez, R. E. Woods and S. L. Eddins, Digital image processing using MATLAB, Pearson Education India, 2004.

[7] N. M. Zaitoun and M. J. Aqel, "Survey on image segmentation techniques," Procedia Computer Science, vol. 65, pp. 797-806, 2015.

[8] N. R. Pal and S. K. Pal, "A review on image segmentation techniques," Pattern recognition, vol. 26, pp. 1277-1294, 1993.

[9] W. Burger and M. J. Burge, Principles of Digital Image Processing: Advanced Methods, Springer Science \& Business Media, 2013.

[10] S. Yuheng and Y. Hao, "Image segmentation algorithms overview," arXiv preprint arXiv:1707.02051, 2017.

[11] H. Gao, W.-C. Siu and C.-H. Hou, "Improved techniques for automatic image segmentation," IEEE transactions on Circuits and Systems for Video Technology, vol. 11, pp. 1273-1280, 2001.

[12] B. Wang, X. Gao, D. Tao and X. Li, "A unified tensor level set for image segmentation," IEEE Transactions on Systems, Man, and Cybernetics, Part B (Cybernetics), vol. 40, pp. 857-867, 2009. 